\title{
Algorithm Refinement for the Stochastic Burgers' Equation
}

\author{
John B. Bell, Jasmine Foo, Alejandro L. Garcia \\ Center for Computational Sciences and Engineering \\ Lawrence Berkeley National Laboratory \\ Berkeley, California, 94720, USA
}

\begin{abstract}
In this paper, we develop an algorithm refinement (AR) scheme for an excluded random walk model whose mean field behavior is given by the viscous Burgers' equation. AR hybrids use the adaptive mesh refinement framework to model a system using a molecular algorithm where desired while allowing a computationally faster continuum representation to be used in the remainder of the domain. The focus in this paper is the role of fluctuations in the dynamics. In particular, we demonstrate that it is necessary to include a stochastic forcing term in Burgers' equation to accurately capture the correct behavior of the system. The conclusion we draw from this study is that the fidelity of multiscale methods that couple disparate algorithms depends on the consistent modeling of fluctuations in each algorithm and on a coupling, such as algorithm refinement, that preserves this consistency.
\end{abstract}




\section{Introduction}

Algorithm Refinement (AR) is an emerging paradigm in the modeling and simulation of multiscale problems. Mathematical models use distinctly different representations for microscopic and macroscopic scales with the corresponding algorithms echoing this disparity. Particle-based algorithms are a class of methods, typically used to model the microscopic scale, that represent the physical system by discrete, interacting entities. These "particles" represent anything from individual atoms to parcels of fluid to bacteria to automobiles. Field-based algorithms, typically used to model the macroscopic scale, are derived from models based primarily on partial differential equations with the physical system represented by continuum fields.

Algorithm Refinement schemes (sometimes called "multi-algorithm hybrids") couple structurally different computational schemes such as particlebased molecular simulations with continuum partial differential equation (PDE) solvers. ${ }^{1}$ The general idea is to perform detailed calculations using an accurate but expensive algorithm in a small region (or for a short time), and couple this computation to a simpler, less expensive method applied to the rest. The formulation of an AR scheme requires: projecting from the microscopic model to macroscopic; refining from macroscopic to microscopic; and handshaking between the two representations where they are coupled. A related issue is the establishment of "refinement criteria" that specify when a microscopic representation is needed and when a macroscopic representation is sufficient. Examples of Algorithm Refinement applied to fluid dynamics may be found in $[13,15,27,32,33,36]$; AR hybrids for interfacial propagation are discussed in $[25,26,28]$.

One aspect of multiscale modeling that has received insufficient attention is the presence of spontaneous fluctuations at microscopic scales and their effect on the macroscopic scale. Accurate modeling of many phenomena require the correct representation of the variances and correlations of fluctuations, specifically when studying systems where the microscopic stochastics drive a macroscopic phenomenon. For physical systems, the correct treatment of fluctuations is especially important for stochastic, nonlinear systems, such as those undergoing phase transitions, nucleation, noise-driven instabilities, combustive ignition, etc.. In these and related applications, the nonlinearities can exponentially amplify the influence of the fluctuations.

Stochastic fluctuations in AR schemes have been investigated for two

\footnotetext{
${ }^{1}$ Note that other types of AR hybrids exist (e.g. coupling spectral and discrete algorithms [31]).
} 
simple diffusive systems: linear diffusion $[3,35]$ and the quasi-linear train model [5]. For those parabolic problems, one finds that when a particle algorithm is coupled to a deterministic continuum algorithm the variance of fluctuations is reduced in the particle regime near the interface. The variance of fluctuations within the continuum regime falls quickly away from the interface; however, variables, such as fluid velocity in the train model, that have long-range correlations retain these correlations of fluctuations (though at reduced magnitude) within the deterministic continuum region. Finally, stochastic continuum algorithms may be formulated such that when coupled to particle schemes they correctly duplicate the physical fluctuations throughout the computational domain.

Our longer term goal is to extend the development of AR methods with fluctuations to an adaptive mesh and algorithm method for the fluctuating compressible Navier-Stokes using a framework analogous to the nonfluctuating CNS solver discussed in [15]. As a prelude to that extension, in the present work we develop an AR method for Burgers' equation that couples nonlinear hyperbolic waves and diffusion. For the particle (microscopic) model we consider the asymmetric excluded random walk (AERW) [23, 30]. The hydrodynamic (macroscopic) model for the AERW model is the viscous Burgers' equation with stochastic forcing $[8,9,11,18]$.

In the next section, we describe in detail the AERW model. In the following section, we introduce the form of Burgers' equation that represents the hydrodynamic limit of the AERW model and describe a discretization of that equation based on a second-order Godunov scheme. In section 4, we discuss the construction of the hybrid method that uses an overall adaptive mesh refinement framework to design the coupling between microscopic and macroscopic models. Section 5 contains computational examples that illustrate and validate the hybrid algorithm. As discussed in the concluding section, the numerical results demonstrate the importance and challenge of accurately modeling fluctuations to simulate and resolve both microscopic and macroscopic phenomena.

\section{Asymmetric Excluded Random Walk}

\subsection{Theory}

The microscopic model for our system is an asymmetric excluded random walk. The AERW model is a system of $N$ random walker particles on a two dimensional rectangular lattice of dimensions $M_{x} \times M_{y}$. Each site is denoted by a coordinate pair $\left(x_{j}, y_{k}\right)$ where $j=1, \ldots, M_{x}$ and $k=1, \ldots, M_{y}$. 
Only one particle may occupy a site; the occupation number $n(x, y)=1$ ( or $=0$ ) if a site is occupied (or unoccupied). We choose the horizontal, or $x$-dimension of the lattice to correspond to the spatial domain of the PDE and define the corresponding density,

$$
u_{p}\left(x_{j}\right)=\frac{1}{M_{y}} \sum_{k=1}^{M_{y}} n\left(x_{j}, y_{k}\right)
$$

so $0 \leq u_{p}(x) \leq 1$. At equilibrium $u_{p}$ is homogeneous and binomially distributed as the sum of $M_{y}$ Bernoulli random variables, each with probability $U=N / M_{x} M_{y}$ of occupation. The mean and variance are,

$$
\left\langle u_{p}\right\rangle=U, \quad\left\langle\delta u_{p}^{2}\right\rangle=\left\langle u_{p}^{2}\right\rangle-\left\langle u_{p}\right\rangle^{2}=\frac{U(1-U)}{M_{y}}
$$

In the local equilibrium approximation the equal-time correlation of fluctuations is

$$
\left\langle\delta u_{p}\left(x_{i}\right) \delta u_{p}\left(x_{j}\right)\right\rangle=\frac{1}{M_{y}}\left\langle u_{p}(x)\right\rangle\left(1-\left\langle u_{p}(x)\right\rangle\right) \delta_{i, j}
$$

At a nonequilibrium steady state the variance is more complicated due to long-ranged correlations of fluctuations [29].

Particles on the lattice move between adjacent sites according to the asymmetric exclusion process. Each particle waits a random time between moves with a mean free time of $\tau$. The next particle to move is drawn at random by choosing a random site, $\left(x_{j}, y_{k}\right)$; if the site is occupied then its particle is selected otherwise another random site is chosen.

The selected particle may move up, down, left or right to an adjacent site, according to the probabilities assigned to the system. We take the particles to move horizontally or vertically with equal probability $\left(p_{\uparrow}=p_{\leftrightarrow}=1 / 2\right)$ and the probabilities of moving up or down conditioned on vertical movement as equal $\left(p_{\uparrow}=p_{\downarrow}=1 / 2\right)$. Asymmetry is introducing by taking unequal conditional probabilities for attempting to move left or right, that is, $p_{\leftarrow} \neq$ $p_{\rightarrow}$ with $p_{\leftarrow}+p_{\rightarrow}=1$. Once the particle and move direction are chosen, the particle moves to the destination site, if unoccupied; if the destination site is occupied then the particle remains in place. In either case the time is advanced and the entire process repeats.

\section{$2.2 \quad$ Numerics}

Given an initial density distribution $u(x)$, the lattice is initialized by randomly filling sites. The dynamics is advanced by randomly choosing 
particles and move directions, as described above. In particle simulations the physical time may be advanced continuously (e.g., event-driven dynamics) or in time increments (e.g., molecular dynamics) and either approach may be used for the AERW. For the former, the time between moves is chosen as an exponential random variable with mean $\tau / N$, where $N$ is the number of particles. For the latter, the number of moves that occur during a time increment $\Delta t_{p}$ is a Poisson distributed random value with mean $\mu=N \Delta t_{p} / \tau$; if $\Delta t_{p} \ll \tau / N$ then the probability of a move occurring during a particle time step $\Delta t_{p}$ is $\mu+O\left(\mu^{2}\right)$.

The lattice is periodic in $y$ so that particles attempting to move up from row $M_{y}$ move to the bottom (first) row, provided it is unoccupied, with a similar definition for particles at the bottom row attempting to move downward. If the $x$-direction is also periodic, then its treatment is analogous to the treatment of periodicity in $y$.

The other type of boundary condition we consider is the imposition of Dirichlet conditions in $x$; in particular, fixing particle densities, $u_{L}$ and $u_{R}$ at the left and right boundaries, respectively. These boundary conditions represent the occupation probabilities for each site on the boundary. We view the system as being augmented with fictitious columns at $j=0$ and $j=M_{x}+1$ and with an effective total number of particles

$$
N_{e}=N+u_{L} M_{y}+u_{R} M_{y} .
$$

We then view the AERW as occurring on the enlarged lattice $\left(\left(M_{x}+2\right) \times M_{y}\right)$ with probabilistic "virtual" particles in the two boundary columns. Operationally these virtual particles enter the algorithm in two ways. First, suppose the selected particle location for the next move is in the left boundary column, say $\left(x_{0}, y_{k}\right)$; with probability $u_{L}$ that site is considered occupied by a virtual particle. If the adjacent site, $\left(x_{1}, y_{k}\right)$, is unoccupied then with probability $p_{\leftrightarrow} p_{\rightarrow}$ a virtual particle moves to that destination, becoming a real particle. Similarly, if a particle attempts to jump into the left boundary from an interior position, the destination is unoccupied with probability $1-u_{L}$ in which case the jump is accepted and the particle removed. Analogous rules apply to the right boundary.

\section{Burgers' equation and continuum method}

\subsection{Theory}

The AERW model of the previous section is defined entirely in terms of a discrete lattice. In order to define a macroscopic model, we spatially 
embed the AERW model by assigning a spatial width $\Delta x_{p}$ to the lattice sites in the $x$ direction. With this definition the hydrodynamic limit of the asymmetric excluded random walk described in the previous section is the stochastic Burgers' equation: [18]

$$
\frac{\partial}{\partial t} u(x, t)=-\frac{\partial}{\partial x}\{f(u)+d(u)+g(x, t)\}
$$

where $g$ is a stochastic flux and

$$
f(u)=c u(1-u) ; \quad d(u)=-\epsilon \frac{\partial u}{\partial x}
$$

are the nonlinear advective flux (sound speed, $\boldsymbol{c}$ ) and the diffusion flux (diffusion constant, $\boldsymbol{\epsilon}$ ). With the change of variable $u^{\prime}=(1-2 u) \boldsymbol{c}$ this may be written in the more traditional form,

$$
u_{t}^{\prime}+u^{\prime} u_{x}^{\prime}=\epsilon u_{x x}^{\prime}+2 c g_{x}
$$

Note that variants of the stochastic Burgers' equation, with different types of stochastic forcing, are common in the literature (e.g., [8]). Also note that there are other particle models, such as the Boghosian-Levermore cellular automaton, that also converge to a stochastic Burgers' PDE in the hydrodynamic limit [10, 22].

The wave speed and diffusion constant are determined from the AERW parameters as,

$$
\boldsymbol{c}=\frac{2 p_{\leftrightarrow} \Delta x_{p}}{\tau}\left(p_{\rightarrow}-\frac{1}{2}\right)=\boldsymbol{c}_{0}\left(2 p_{\rightarrow}-1\right)
$$

and

$$
\boldsymbol{\epsilon}=\frac{2 p_{\leftrightarrow} \Delta x_{p}^{2}}{\tau} p_{\rightarrow} p_{\leftarrow}=2 \boldsymbol{c}_{0} \Delta x_{p} p_{\rightarrow}\left(1-p_{\rightarrow}\right)
$$

where $\boldsymbol{c}_{0}=p_{\leftrightarrow} \Delta x_{p} / \tau$ is the wave speed for the completely asymmetric walk $\left(p_{\rightarrow}=0\right.$ or 1$)$. Since the wave speed $f^{\prime}(u)$ varies between $+c$ and $-c$ on the range of $u$, we define a dimensionless cell Reynolds number as

$$
\operatorname{Re}_{c}=\frac{|\boldsymbol{c} \Delta x|}{\boldsymbol{\epsilon}}=\frac{\left|p_{\rightarrow}-\frac{1}{2}\right|}{p_{\rightarrow}\left(1-p_{\rightarrow}\right)}
$$

that characterizes the relative importance of diffusion and advection for the dynamics at a given mesh spacing. Note that for $p_{\rightarrow}=1 / 2$ the random walk is symmetric (pure diffusion) and $\operatorname{Re}_{c}=0$; as $p_{\rightarrow}$ approaches 0 or 1 the random walk is unidirectional (pure advection) and $\operatorname{Re}_{c}$ goes to infinity. 
The stochastic flux is a Gaussian white noise with zero mean and correlation

$$
\left\langle g(x, t) g\left(x^{\prime}, t^{\prime}\right)\right\rangle=A(x, t) \delta\left(x-x^{\prime}\right) \delta\left(t-t^{\prime}\right)
$$

where the brackets denote ensemble average. The noise amplitude, $A(x, t)$, is related the correlation of density fluctuations; in the local equilibrium approximation,

$$
\left\langle\delta u(x, t) \delta u\left(x^{\prime}, t\right)\right\rangle=\hat{u}(x, t)(1-\hat{u}(x, t)) \delta\left(x-x^{\prime}\right)
$$

where $\hat{u}$ is the solution to the deterministic Burgers' equation, that is,

$$
\hat{u}_{t}=-f(\hat{u})_{x}+\epsilon \hat{u}_{x x}
$$

From the above one finds,

$$
A(x, t)=2 \epsilon \hat{u}(1-\hat{u}) .
$$

The noise amplitude may also be obtained from the continuum limit of the master equation for the AERW [18].

\section{$3.2 \quad$ Numerics}

The stochastic Burgers' equation may be simulated numerically by a variety of CFD algorithms, with the choice guided by the application. For example, spectral methods have been developed for homogeneous, isotropic turbulence (see $[19,24]$ and references therein). For our AR hybrid we choose a cell-centered finite difference method, specifically a second-order Godunov scheme to calculate the hyperbolic flux and a simple explicit predictorcorrector centered difference scheme to compute the diffusion term. We denote the spatial and temporal grid sizes as $\Delta x$ and $\Delta t$ and denote by $u_{j}^{n}$ the average of $u$ in cell $-j$ at time $n$.

The second-order Godunov scheme constructs a linear profile within each cell with the slopes estimated by a higher-order finite difference approximation

$$
u_{x, j}=\frac{-u_{j+2}^{n}+8 u_{j+1}^{n}-8 u_{j-1}^{n}+u_{j-2}^{n}}{12 \Delta x}
$$

For advection-dominated problems a limiter is typically applied to these slopes; however, in the present context, we are resolving at the viscous length scale so no limiting is performed. These slopes are used to predict values at cell interfaces at the half-time level $t^{n+1 / 2}$. In particular, we define

$$
u_{j+1 / 2, \ell}^{n+1 / 2}=u_{j}^{n}+\frac{1}{2}\left[\Delta x-\Delta t \max \left(f^{\prime}\left(u_{j}^{n}\right), 0\right)\right] u_{x, j}
$$


and

$$
u_{j+1 / 2, r}^{n+1 / 2}=u_{j+1}^{n}-\frac{1}{2}\left[\Delta x+\Delta t \min \left(f^{\prime}\left(u_{j}^{n}\right), 0\right)\right] u_{x, j}
$$

where $f^{\prime}=d f / d u$. We then define the hyperbolic flux $f_{j+1 / 2}^{n+1 / 2}=f\left(u_{j+1 / 2}^{n+1 / 2}\right)$ where $u_{j+1 / 2}^{n+1 / 2}$ is the solution of the Riemann problem for $u_{t}+f_{x}=0$ along the ray $x / t=0$ with left and right states $u_{j+1 / 2, \ell}^{n+1 / 2}$ and $u_{j+1 / 2, r}^{n+1 / 2}$, respectively.

The diffusion and stochastic flux terms are evaluated using a predictorcorrector scheme, treating the hyperbolic flux terms as source terms. In particular, we first compute predicted values

$$
\begin{aligned}
u_{j}^{p}= & u_{j}^{n}-\frac{\Delta t}{\Delta x}\left(f_{j+1 / 2}^{n+1 / 2}-f_{j-1 / 2}^{n+1 / 2}\right) \\
& +\frac{\Delta t}{(\Delta x)^{2}} \epsilon\left(u_{j-1}^{n}-2 u_{j}^{n}+u_{j+1}^{n}\right)+\frac{\sqrt{2} \Delta t}{\Delta x}\left(g_{j+1 / 2}^{n}-g_{j-1 / 2}^{n}\right)
\end{aligned}
$$

We then compute corrected values

$$
\begin{aligned}
u_{j}^{n+1}= & \frac{u_{j}^{p}}{2}+\frac{1}{2}\left(u_{j}^{p}-\frac{\Delta t}{\Delta x}\left(f_{j+1 / 2}^{n+1 / 2}-f_{j-1 / 2}^{n+1 / 2}\right)\right. \\
& \left.+\frac{\Delta t}{(\Delta x)^{2}} \epsilon\left(u_{j-1}^{p}-2 u_{j}^{p}+u_{j+1}^{p}\right)+\frac{\sqrt{2} \Delta t}{\Delta x}\left(g_{j+1 / 2}^{p}-g_{j-1 / 2}^{p}\right)\right)
\end{aligned}
$$

This can be rewritten as,

$$
u_{j}^{n+1}=u_{j}^{n}-\frac{\Delta t}{\Delta x}\left(F_{j+1 / 2}^{n}-F_{j-1 / 2}^{n}\right)
$$

where,

$$
F_{j+1 / 2}^{n}=f_{j+1 / 2}^{n+1 / 2}-\frac{1}{2}\left(\epsilon \frac{u_{j+1}^{n}-u_{j}^{n}}{\Delta x}+\epsilon \frac{u_{j+1}^{p}-u_{j}^{p}}{\Delta x}\right)-\frac{1}{\sqrt{2}}\left(g_{j+1 / 2}^{n}+g_{j+1 / 2}^{p}\right)
$$

is the total flux.

The stochastic flux for the asymmetric excluded random walk on an $M_{x} \times M_{y}$ lattice is discretized as,

$$
g_{j+1 / 2}^{n, p}=\sqrt{\frac{A_{j}^{n, p}+A_{j+1}^{n, p}}{2 \Delta t M_{y}}} \Re .
$$

where

$$
A_{j}^{n, p}=2 \epsilon \tilde{u}_{j}^{n, p}\left(1-\tilde{u}_{j}^{n, p}\right), \quad \tilde{u}=\min (\max (u, 0), 1)
$$


and $\Re$ is a Gaussian (normal) distributed random variable. Note that the instantaneous fluctuating values are used in place of the deterministic value (see eqn. 14), which is accurate as long as the fluctuations remain small. [16].

The scheme outlined above is stable provided it satisfies time step limits for both the hyperbolic and diffusive terms. In particular, we require

$$
\frac{\Delta t \max \left|f^{\prime}(u)\right|}{\Delta x} \leq 1 \quad \text { and } \quad \frac{\epsilon \Delta t}{\Delta x^{2}} \leq \frac{1}{2}
$$

We also note that it is possible to use a simpler version of the scheme based on an explicit first-order treatment of the diffusion term. For the most part, the simpler version provides reasonable predictions; however, at larger $\Delta t$ the first-order scheme over-predicts the variation in the equilibrium solution by approximately $5 \%$, suggesting that the temporal truncation error terms suppress the smoothing effect of the diffusion.

\section{Algorithm Refinement Hybrid}

In this section we develop a hybrid algorithm refinement method that couples the AERW model introduced in section 2 with the stochastic Burgers' equation algorithm in section 3 .

\subsection{Basic construction}

Philosophically, the construction of the hybrid is based on the notion that the particle description provides a more accurate representation of the solution than the stochastic PDE. Thus the basic idea is to represent the dynamics with the continuum model except in a localized region where higherfidelity particle representation is required.

Our perspective in designing the algorithm follows the adaptive mesh and algorithm (AMAR) approach introduced in [15]. In contrast to other AR approaches (see, e.g., [13]), the AMAR approach maintains a solution of the macroscopic model over the entire domain (see Fig. 1). An error estimation criterion is used to estimate where the improved-representation of the particle method is required. That region, which can change dynamically, is then "covered" with a particle patch. In this hierarchical representation the solution is given by the particle solution on the region covered by the particle patches and the continuum solution on the remainder of the domain.

The coupling between the particle and continuum regions uses the ana$\log$ of constructs used in developing hierarchical adaptive mesh refinement algorithms. For simplicity we will assume that there is a single refined patch 
and that the mesh spacing for the continuum solver is equal to the lattice spacing $\Delta x_{p}$. Generalization of the approach to include multiple patches (e.g. [37]) and allowing the continuum mesh to be an integral multiple of $\Delta x_{p}$ (e.g., [4]) is fairly straightforward.

Integration on the hierarchy is a three step process. First, we integrate the continuum algorithm from $t^{n}$ to $t^{n+1}$, i.e., for a continuum step $\Delta t$. The old and new states, $u_{j}^{n}$ and $u_{j}^{n+1}$, are retained until the particle time step is complete. Continuum data at the edge of the particle patch is interpolated in time to provide Dirichlet boundary conditions for the particle method.

We have considered both of the time-evolution schemes discussed in section 2. For the equal time step version, we choose $\Delta t_{p}$ so that $\Delta t_{p}=\Delta t / M_{t}$ for a specified integer $M_{t}$. We then advance the particle method by $M_{t}$ steps until the particle and the continuum solver are at the same time. For the random time version of the algorithm, the particle method is advanced by moves, each with a random time increment, until the next transition would advance the particle time beyond $t^{n+1}$ at which point the two solutions are synchronized. We note that at the synchronization juncture the particle and continuum solutions are not quite at the same time level. For the most part, this has little effect on the computational results; however, it does lead to errors of approximately $1 \%$ in the mean solution at equilibrium, which is not observed with the temporally synchronized version.

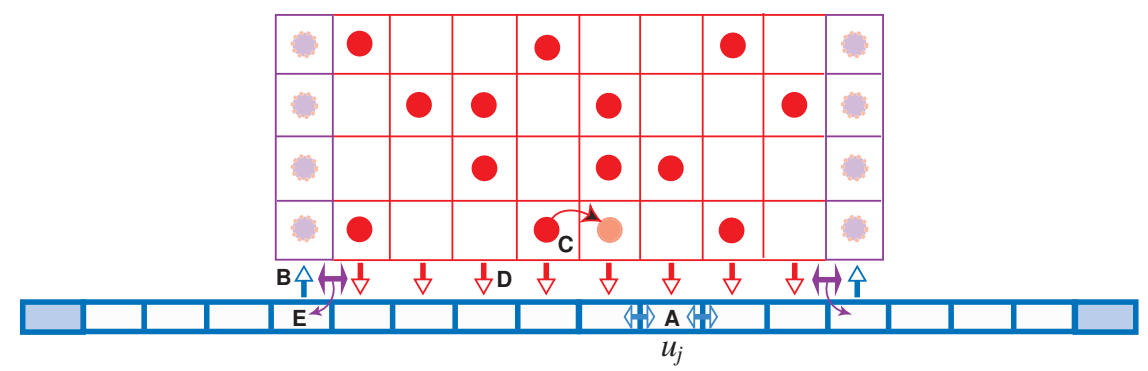

Figure 1: Schematic of AERW/PDE hybrid: (A) Advance continuum solution; (B) Set boundary conditions for AERW from time-interpolated PDE solution; (C) Advance particle system by AERW. Synchronize solutions: (D) Replace overlaying continuum values with particle values; (E) Reset PDE interface cells by refluxing. 


\subsection{Synchronization}

The initial stage of the integration process essentially advances the macroscopic model separately with a one-way coupling to the microscopic model by way of the Dirichlet boundary conditions. The macroscopic model is not influenced by the microscopic model; the goal of the synchronization process is to correct the macroscopic solution to reflect the effect of the microscopic model as though the integration were tightly coupled.

There are two components to the synchronization process. First, on the region covered by the particle representation we replace the continuum solution obtained from the SPDE discretization by the more accurate particle representation, i.e, set

$$
u_{j}^{n+1}=\frac{1}{M_{y}} \sum_{k=1}^{M_{y}} n\left(x_{j}, y_{k}\right)
$$

for each cell covered by the particle patch. Second, the continuum cells immediately adjacent to the particle region, which supplied boundary data for the particle region during its advance, are corrected by "refluxing." Specifically, suppose the left boundary of the particle patch occurs in cell $J+1$. The value in continuum cell $J$ was updated with the continuum scheme

$$
u_{J}^{n+1}=u_{J}^{n}-\frac{\Delta t}{\Delta x}\left(F_{J+1 / 2}^{n}-F_{J-1 / 2}^{n}\right)
$$

with the fluxes $F^{n}$ computed from the continuum values (see eqn. (20)). The microscopically correct flux is given by the net number of particles moving across edge $J+1 / 2$ rather than by the continuum flux $F_{J+1 / 2}^{n}$. To perform the refluxing correction we monitor the number of particles, $N_{J+1 / 2}$ and $N_{J+1 / 2}^{\leftarrow}$, that move into and out of the particle region, respectively, across the continuum/particle interface at edge $J+1 / 2$. We then correct the continuum solution as

$$
u_{J}^{\prime n+1}=u_{J}^{n+1}+\frac{\Delta t}{\Delta x} F_{J+1 / 2}^{n}-\frac{N_{J+1 / 2}^{\rightarrow}-N_{J+1 / 2}^{\leftarrow}}{\Delta x M_{y}}
$$

This update effectively replaces the continuum flux component of the update to $u_{J}^{n+1}$ on edge $J+1 / 2$ by the flux of particles through the edge. An analogous refluxing step occurs in the cell adjacent to the right hand boundary of the particle region. Finally, note that this synchronization procedure guarantees exact conservation of integrated density. 


\subsection{Refinement criterion and regridding}

The AMAR framework allows us to dynamically change the location of the particle region. There are several possible strategies for designing refinement criteria. For the examples described in section 5.3, we will focus on criteria that identify cells where the solution has a large gradient characteristic of a viscous shock profile. A straightforward measurement of the local gradient of the solution (e.g., $\left.\left(u_{j+1}-u_{j}\right) / \Delta x\right)$ is not adequate since the inherent fluctuations could trigger refinement even at equilibrium. What is needed is a robust measure that identifies viscous shocks without generating substantial "false positives" leading to unnecessary refinement. To this end we define a regional gradient using

$$
D_{j}=\frac{1}{S \Delta x}\left[\frac{1}{S} \sum_{i=1}^{S} u_{j+i}-\frac{1}{S} \sum_{i=1}^{S} u_{j-(i-1)}\right]=\sum_{i=1}^{S} \frac{u_{j+i}-u_{j+1-i}}{S^{2} \Delta x}
$$

where the stencil size $S$ is specified; we take $S=4$ in the computations in the next section. From eqn. (3) one may easily estimate the expected standard deviation $\sigma$ of $D$ resulting from equilibrium fluctuations and set a tolerance of $C \sigma$ where $C$ is a constant. To estimate where to place the particle region in the adaptive code we compute the regional gradient at each point; if $\left|D_{j}\right|$ exceeds the tolerance level, then cells $j$ and $j+1$ are tagged. Since we restrict ourselves to a single particle patch, the largest interval containing all tagged cells is then the new particle region. If multiple patches were allowed then tagged cells would be collected to form particle regions; techniques for collecting tagged cells in an optimal manner are well established in the mesh refinement literature [7].

Once the new particle region has been identified, it must be initialized. For continuum cells that were already in the particle region, we simply retain the distribution of occupied sites. For cells that were not in the previous particle region, we use the continuum density to compute $N_{f}$, the desired number of particles for filling a column. The simple way to do this is to take $N_{f}=u_{j}^{n} M_{y}$ rounded to the nearest integer; an alternative approach would be to fill randomly each site with probability $u_{j}^{n}$. We use the former approach since it preserves conservation of total density (to within quantization rounding). We note the the regridding algorithm does not need to be done every step. Simple estimates based either on CFL considerations or estimates of discrete traveling wave velocities can be used to determine how often to regrid [6]. 


\section{Computational Examples}

This section presents a series of computational examples, of progressively increasing sophistication, that demonstrate the accuracy and effectiveness of the Algorithm Refinement hybrid. We consider four numerical schemes: the Asymmetric Excluded Random Walk (AERW) from section 2; the stochastic PDE (SPDE) for Burgers' equation from section 3 and two Algorithm Refinement hybrids from section 4 . The first hybrid couples the AERW and SPDE, with the particle scheme in a single patch within the system. The second hybrid is similar but without a stochastic flux, that is, using a deterministic PDE (DPDE). Both fixed-patch and adaptive hybrids are considered as well as a handful of minor variants, discussed below.

The following parameter values are common to the simulations in all the examples: $M_{y}=150, \Delta x=\Delta x_{p}=0.01, \tau=1, p_{\leftrightarrow}=1 / 2, p_{\uparrow}=p_{\downarrow}=1 / 2$, and $\boldsymbol{c}_{0}=0.01$. The particle time step is chosen to be small $\left(\Delta t_{p} \approx \tau /(75 N)\right)$ so the probability of a move occurring during a time step is taken as $N \Delta t_{p} / \tau$. For a system length of $L$ the viscous relaxation time is $T_{\boldsymbol{\epsilon}}=L^{2} / \boldsymbol{\epsilon}$; in our simulations $T_{\boldsymbol{\epsilon}}=O\left(10^{4}\right)$. For the simulations of steady states the system is initialized near the final state and allowed to relax for a time that is long compared to the relaxation time (typically for $>100 T_{\epsilon}$ ) before taking samples.

\subsection{Equilibrium State}

First we consider the simplest scenario, a system at the equilibrium state with equal, fixed density at $x=-0.5$ and 0.5 . The probability of moving to the right is $p_{\rightarrow}=0.55$, corresponding to a cell Reynolds number of $\operatorname{Re}_{c}=0.20$ (weakly hyperbolic). The single-algorithm simulations (AERW and Burger's SPDE) have 100 sites or grid points in the $x$-direction. The AR hybrids introduce a fixed particle patch at the center of the system between $x=-0.1$ and $x=0.1$ with $M_{x}=20$. The hybrid simulation is performed at three continuum time step sizes: $\Delta t=0.05,0.1$, and 0.2 . Since incremental time stepping is used in the particle algorithm, to keep $\Delta t_{p}=\Delta t / M_{t} \ll \Delta t$ we take three corresponding values: $M_{t}=8000,14000$, and 28000. Each simulation is run to a final time of $T=2 \times 10^{7}$, which corresponds to $N_{t}=T / \Delta t$ continuum time steps (e.g., $N_{t}=4 \times 10^{8}$ for the smallest $\Delta t$ ).

Typical results from the various numerical schemes are shown in Fig. 2 where the mean, $\langle u\rangle$; variance, $\left\langle\delta u^{2}\right\rangle$; and correlation, $\left\langle\delta u \delta u^{\prime}\right\rangle$ of density are plotted versus position. These three quantities are estimated from samples 

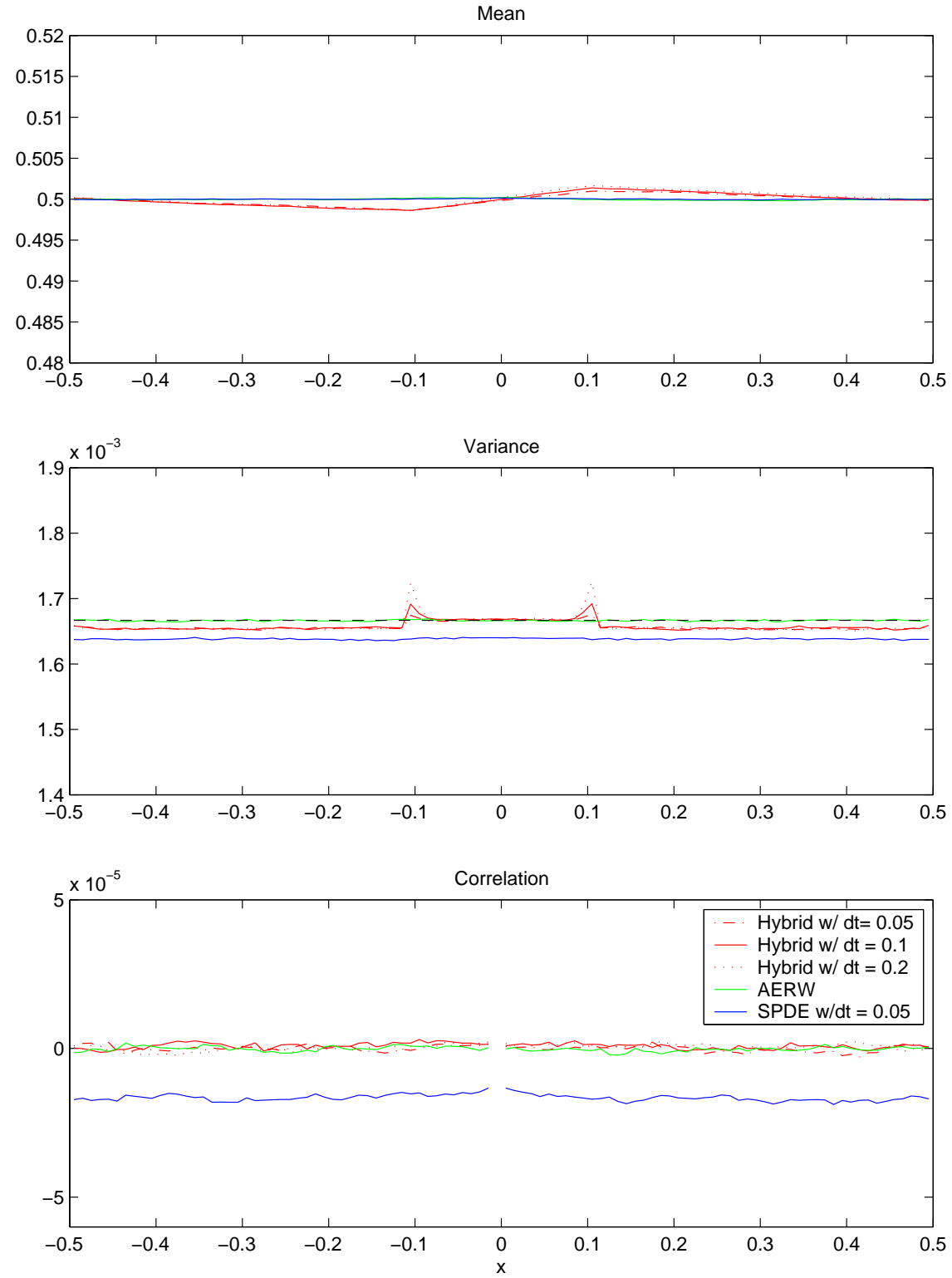

Figure 2: Mean $\langle u\rangle$, variance $\left\langle\delta u^{2}(x)\right\rangle$ and center point correlation $\langle\delta u(x) \delta u(0)\rangle$ versus $x$ for a system at equilibrium $\left(u_{L}=u_{R}=0.5\right)$. Lines are: SPDE/AERW hybrid with $\Delta t=0.05$ (red dashed dot); SPDE/AERW hybrid with $\Delta t=0.1$ (solid red); SPDE/AERW hybrid with $\Delta t=0.2$ (red dotted); AERW (green); SPDE with $\Delta t=0.05$ (solid blue); predicted variance $\langle u\rangle(1-\langle u\rangle) / M_{y}$ (dashed black). Note that for the correlation $\langle\delta u(x) \delta u(0)\rangle$ the large spike at $x=0$ is omitted from the plot. 
as,

$$
\begin{aligned}
\left\langle u\left(x_{i}\right)\right\rangle & =\frac{1}{N_{s}} \sum_{n=N_{r}+1}^{N_{t}} u_{i}^{n} ; \\
\left\langle\delta u\left(x_{i}\right)^{2}\right\rangle & =\left\langle\delta u\left(x_{i}\right) \delta u\left(x_{i}\right)\right\rangle \\
\left\langle\delta u\left(x_{i}\right) \delta u\left(x_{j}\right)\right\rangle & =\frac{1}{N_{s}} \sum_{n=N_{r}+1}^{N_{t}} u_{i}^{n} u_{j}^{n}-\left\langle u\left(x_{i}\right)\right\rangle\left\langle u\left(x_{j}\right)\right\rangle
\end{aligned}
$$

where $N_{s}=N_{t}-N_{r}$ is the number of samples and $N_{r}$ is the number of continuum time steps that the system is allowed to relax before sampling begins.

Even at equilibrium the stochastic PDE scheme does not exactly match the AERW results, for example the SPDE method has an error of about $1.8 \%$ in the variance and a relative error in the correlation of $\left\langle\delta u \delta u^{\prime}\right\rangle /\left\langle\delta u^{2}\right\rangle \approx 1 \%$. This discrepancy is expected since the numerical scheme uses the instantaneous solution to compute the amplitude of the noise instead of the mean (see eqns. (14) and (23)). This effect is illustrated in Fig. 3, which compares simulations where the mean and instantaneous state are used to calculate the noise amplitude and verifies that the former is in agreement with the AERW results. Obviously the mean is known at equilibrium but for timedependent, non-equilibrium problems the SPDE method needs to use instantaneous values of the state to evaluate the noise. In any case, eqn. (14) is only rigorously valid at equilibrium.

In general the SPDE/AERW hybrid gives good results with a small discrepancy due to the effect just described regarding the noise amplitude. For the hybrid simulations, there is a small error in the mean of approximately $0.3 \%$ of the solution which decreases to about $0.2 \%$ when $\Delta t$ is decreased to 0.05. This error is due to the buildup of the discrepancy between SPDE and AERW models at the boundaries of the particle patch in the hybrid. In the variance, we see an error of about $0.6 \%$ in the SPDE part of the domain, due to the same effect. In addition, there are spikes at the edges of the particle patch, representing an error of $3 \%$ at $\Delta t=0.2$ decreasing to about $0.3 \%$ at $\Delta t=0.05$, evidently due to the temporal truncation error in the SPDE solver.

Figure 4 illustrates the effect on fluctuations when the continuum PDE scheme does not include a stochastic flux. Clearly the variance drops to near zero inside the DPDE regions, left and right of the particle patch, yet the variance within the patch remains nearly correct except near the interface. As discussed in the introduction, this general result was observed 


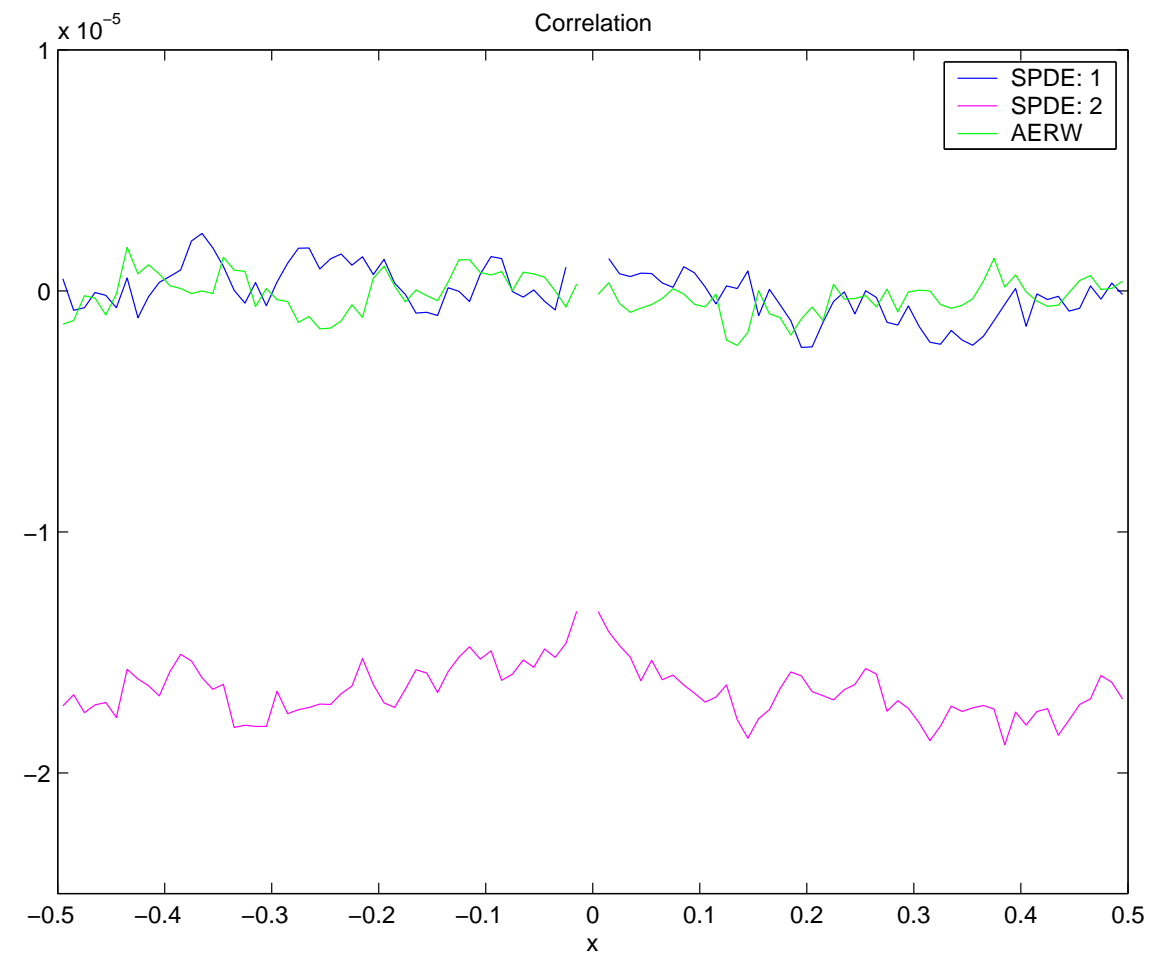

Figure 3: Center point correlation versus $x$ for a system at equilibrium $\left(u_{L}=\right.$ $\left.u_{R}=\langle u\rangle=0.5\right)$ with the SPDE method using mean and instantaneous solution for the noise amplitude. Lines are: SPDE: 1 using the mean (blue); SPDE: 2 using the instantaneous solution (magenta); AERW (green). Time step is $\Delta t=0.05$. 

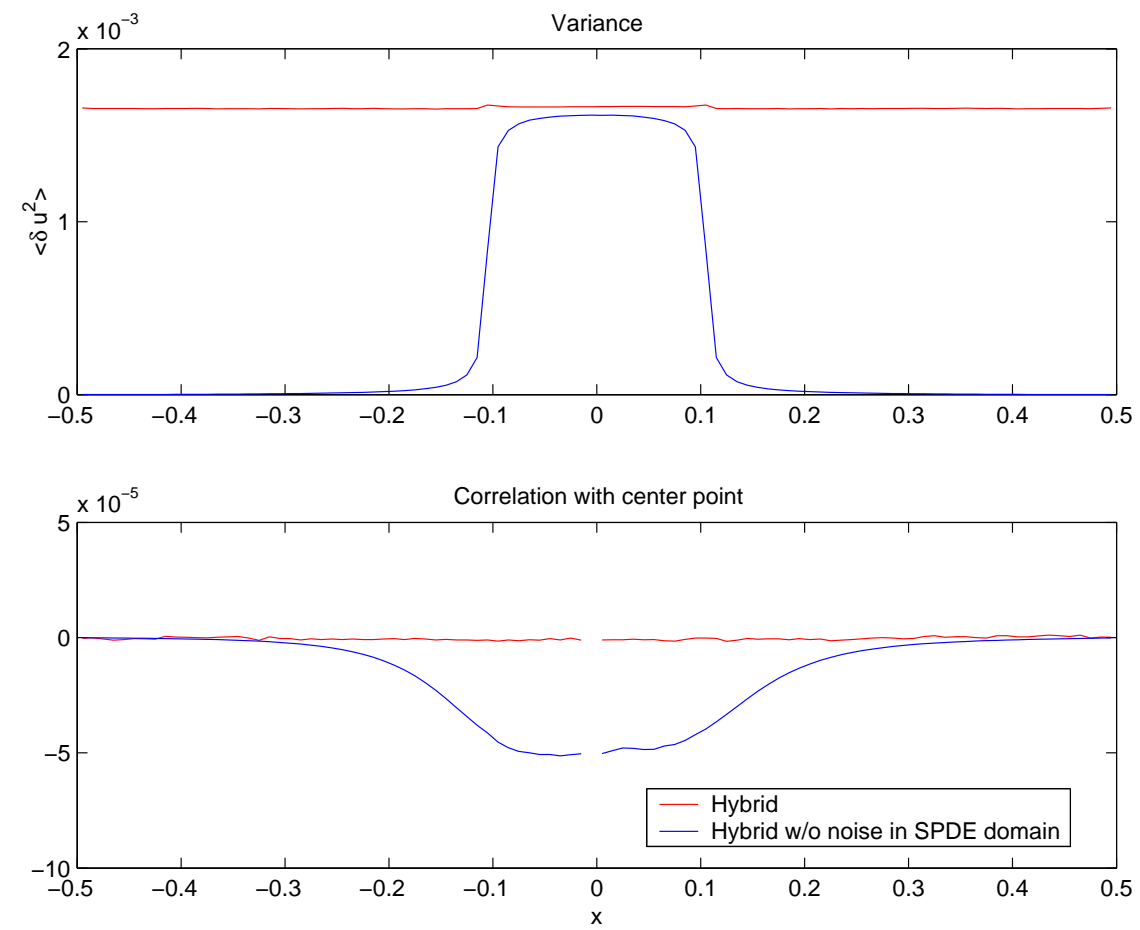

Figure 4: Variance $\left\langle\delta u^{2}(x)\right\rangle$ and center-point correlation $\langle\delta u(x) \delta u(0)\rangle$ versus $x$ for a system at equilibrium $\left(u_{L}=u_{R}=0.5\right)$. Lines are: SPDE/AERW hybrid (red); DPDE/AERW hybrid (blue) (compare with Fig. 2). For both cases $\operatorname{Re}_{c}=0.20\left(p_{\rightarrow}=0.55\right)$ and $\Delta t=0.05$.

in previous studies of AR hybrids for parabolic systems [4, 5] but it was not obvious that hyperbolic systems would be similar. Even more interesting is the appearance of a large correlation of fluctuations in the particle region of the DPDE/AERW hybrid, an effect that will be discussed in the next subsection.

One final note: Using periodic boundary conditions gives similar results to those presented above for Dirichlet boundary conditions. Furthermore, using periodic boundary conditions we confirmed that the AR hybrids conserve total density, $\sum_{i} u_{i}$, exactly. (When the grids move dynamically this exact conservation is lost because of quantization effects in defining a particle distribution from continuum data.) 


\subsection{Rarefaction Steady State}

Next we consider nonequilibrium states by taking different densities at the left and right boundaries. For $c>0$ the steady solutions to Burgers' equation are: a shock wave if $u_{L}<u_{R}$ and a rarefaction wave if $u_{L}>u_{R}$. In this subsection we examine the latter, turning to shocks in the last two examples.

The parameters used for the rarefaction steady state are $u_{L}=0.9, u_{R}=$ $0.1, L=1, \operatorname{Re}_{c}=0.20\left(p_{\rightarrow}=0.55\right), T=4 \times 10^{7}$, and $\Delta t=0.05$. In the hybrid, $M_{t}=8000$ and the particle patch is fixed between $x=-0.1$ and 0.1 .

Figure 5 shows typical results from the simulations. All methods give good agreement with the AERW method in the mean. As in the equilibrium case, the hybrid AERW/SPDE gives good agreement in the variance with both the AERW and SPDE solvers. However, when the AERW is coupled to the deterministic PDE solve, the variance falls to zero outside the particle patch while remaining close to the correct value inside the particle patch (similar to the equilibrium result of Fig. 4).

Figure 5 shows that a long-range correlation, predicted by [12, 17, 29], is observed in the AERW, SPDE, and AERW/SPDE solvers and the three simulations are in good agreement with each other. This figure also shows that the AR hybrid using a deterministic PDE solver erroneously enhances this correlation; Fig. 2 shows a similar effect appearing even at equilibrium, where no correlation is expected. It is not clear why this occurs since the correlation in a related AR hybrid of the "train" model is diminished [5]. One possible explanation is the induction of spurious correlations, even at equilibrium, when a reservoir does not generate the correct fluctuations spectrum [34].

\subsection{Shock Tracking}

Unlike the rarefaction case, when $u_{L}<u_{R}$ the deterministic solution develops a shock wave in finite time propagating with speed $\sigma=\boldsymbol{c}(1-$ $\left.u_{L}-u_{R}\right)$. When viscous terms are added, the solution forms a smooth travelling wave moving at speed $\sigma$. Figures 6,7 , and 8 show examples of propagating shock waves for increasing cell Reynolds number. For these examples we have used the automatic criterion discussed in section 4.3 to localize the particle region around the shock. Note that in each case the refinement criteria does a good job of localizing the particle region near the shock. For the most diffuse case, $p_{\rightarrow}=0.55$, the solution looks essentially 

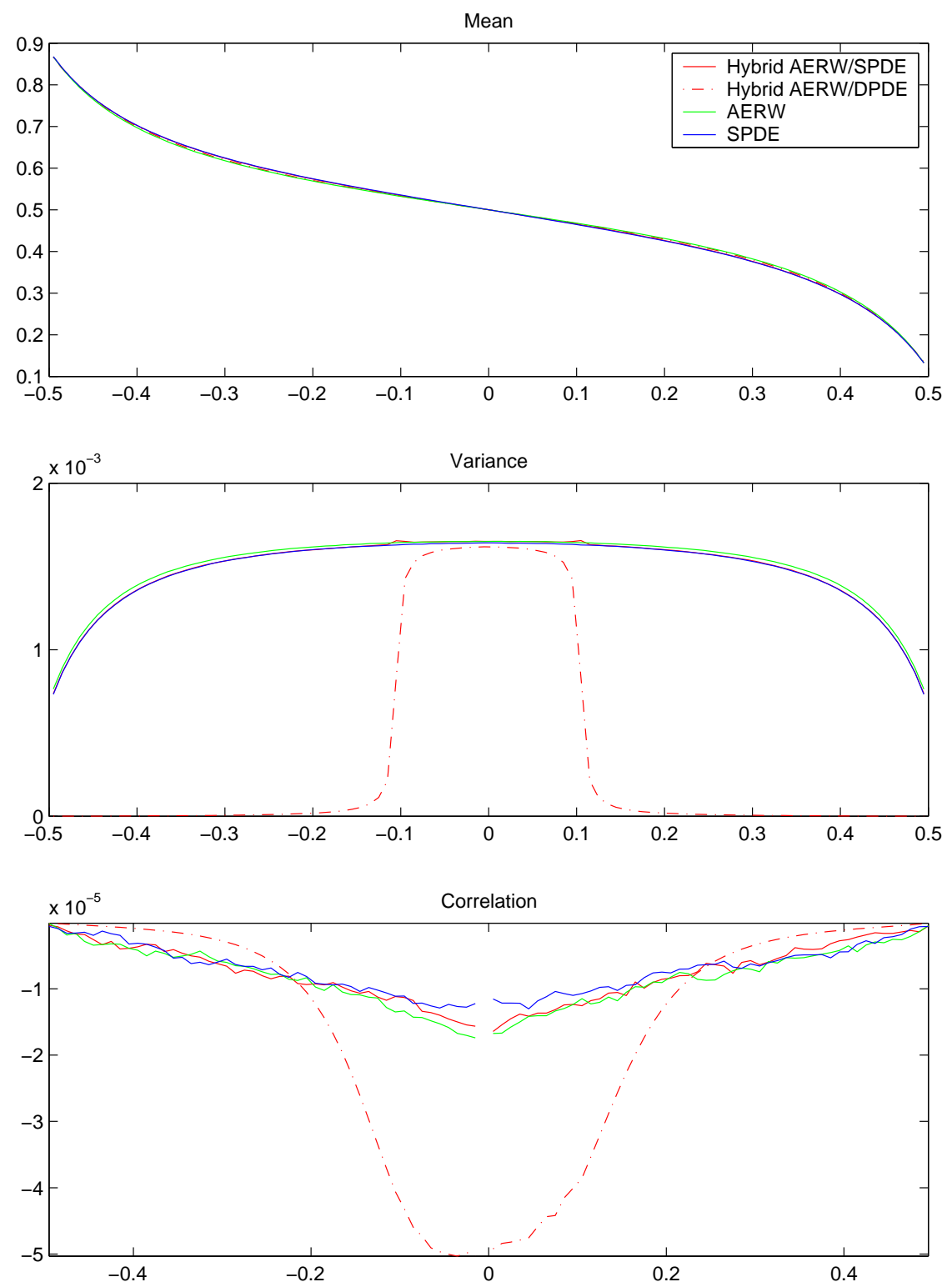

Figure 5: Mean $\langle u\rangle$, variance $\left\langle\delta u^{2}(x)\right\rangle$ and center point correlation $\langle\delta u(x) \delta u(0)\rangle$ versus $x$ for a rarefaction steady state $\left(u_{L}=0.9, u_{R}=0.1\right)$. Lines are: Hybrid AERW/SPDE (red); Hybrid AERW/DPDE (dashed dot red); AERW (green); SPDE (blue). 
like the deterministic solution with superimposed noise. On the other hand, for the stronger shocks the fluctuations introduce sufficient perturbations to noticeably shift the shock location; this drift in the shock position is investigated further in the next subsection.
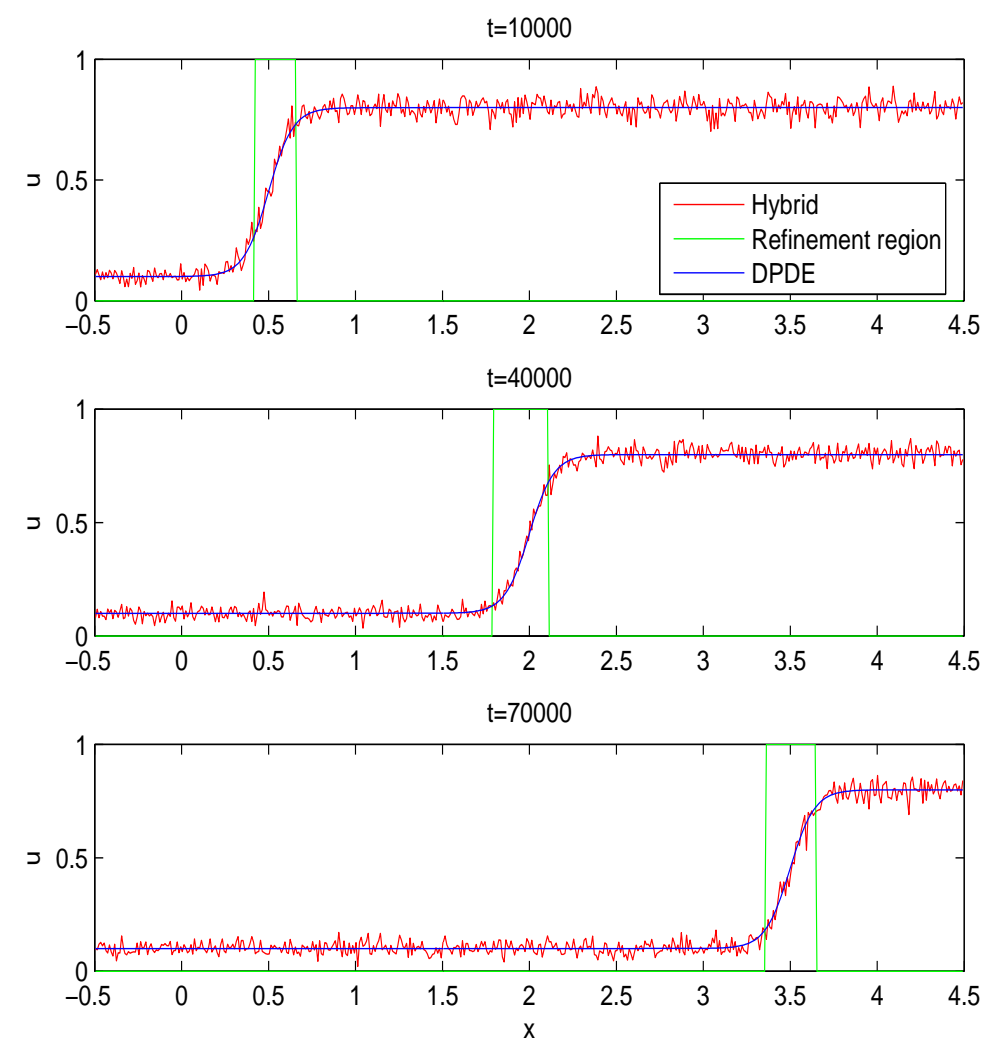

Figure 6: Instantaneous density $u(x, t)$ versus position $x$ for a moving shock $\left(u_{L}=0.1, u_{R}=0.8\right)$ for $\operatorname{Re}_{c}=0.20\left(p_{\rightarrow}=0.55\right)$, which has shock speed $\sigma=5 \times 10^{-5}$. Methods used are: AERW/SPDE hybrid (red); DPDE (blue); vertical green lines delineate the AERW particle region of the hybrid.

\subsection{Shock Diffusion}

Motivated the observations in the previous section regarding the variation in the shock position, we consider the diffusion of the position of a 

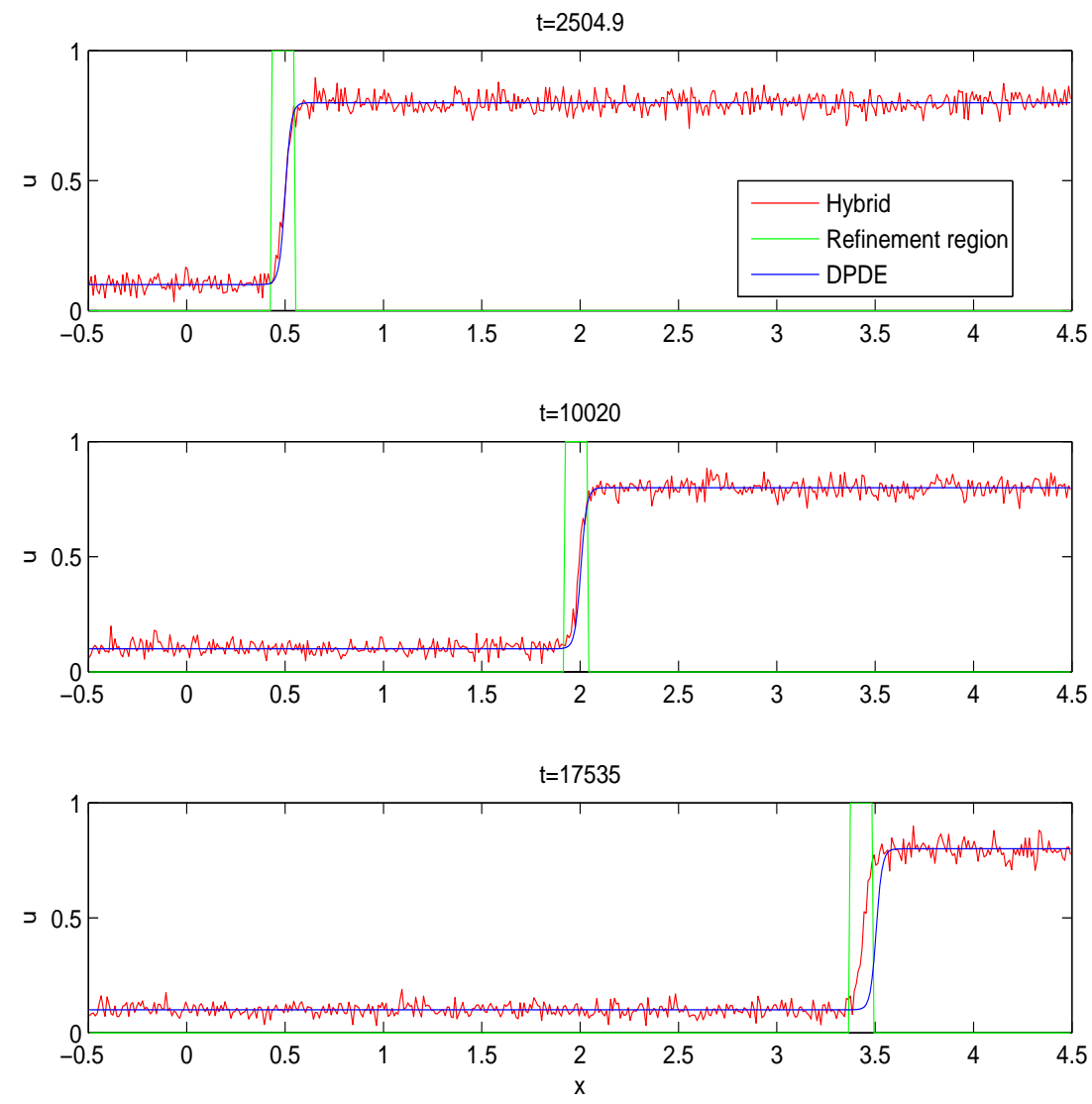

Figure 7: Instantaneous density $u(x, t)$ versus position $x$ for a moving shock $\left(u_{L}=0.1, u_{R}=0.8\right)$ for $\operatorname{Re}_{c}=0.95\left(p_{\rightarrow}=0.7\right)$, which has shock speed $\sigma=2 \times 10^{-4}$. Methods used are: AERW/SPDE hybrid (red); DPDE (blue); vertical green lines delineate the AERW particle region of the hybrid.

stationary shock. The general problem has been analyzed for the AERW and many results are known $[2,1,14,20]$ but here we focus on the variance of the shock location as a function of time. We define a shock location, $s(t)$ by fitting a Heaviside function of equal integrated density, that is,

$$
\int_{-L / 2}^{s(t)} u_{L} d x+\int_{s(t)}^{L / 2} u_{R} d x=\int_{-L / 2}^{L / 2} u(x, t) d x
$$



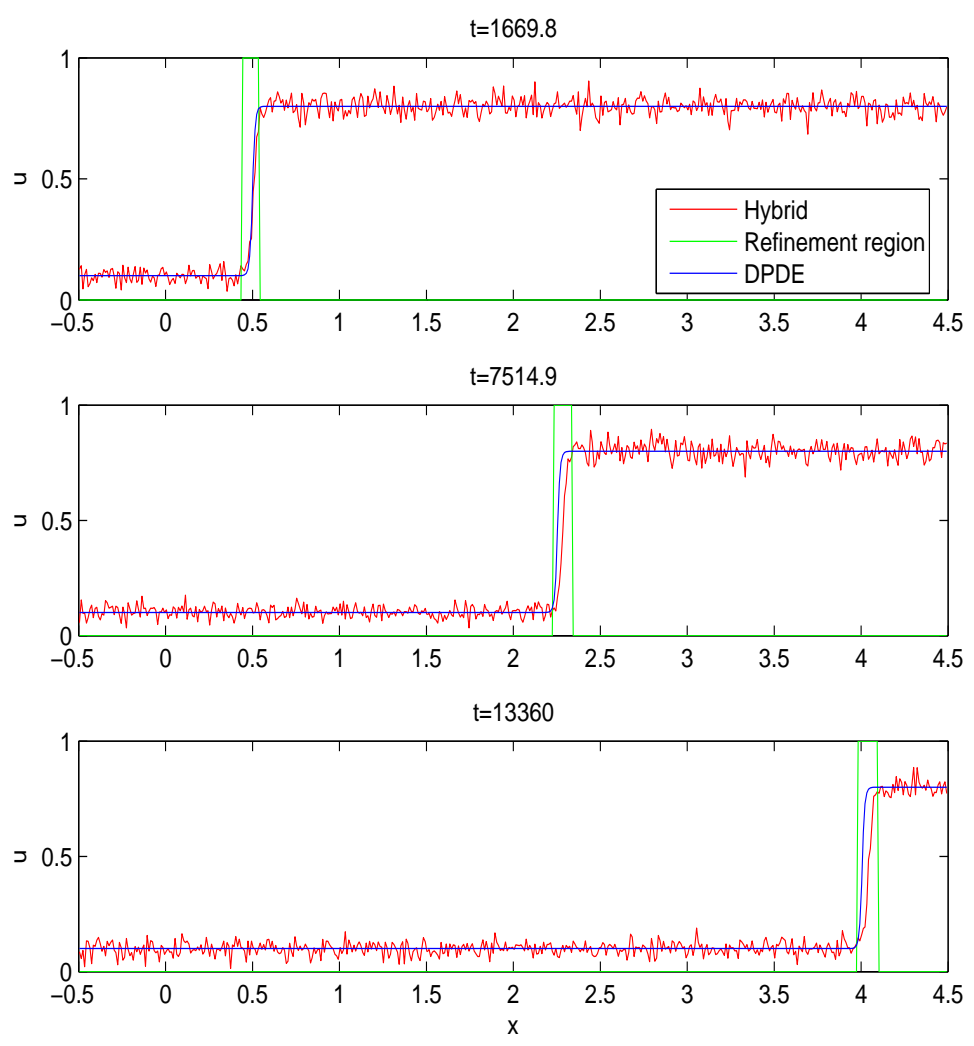

Figure 8: Instantaneous density $u(x, t)$ versus position $x$ for a moving shock $\left(u_{L}=0.1, u_{R}=0.8\right)$ for $\operatorname{Re}_{c}=1.87\left(p_{\rightarrow}=0.8\right)$, which has shock speed $\sigma=3 \times 10^{-4}$. Methods used are: AERW/SPDE hybrid (red); DPDE (blue); vertical green lines delineate the AERW particle region of the hybrid.

from which we find

$$
s(t)=L \frac{\bar{u}(t)-1 / 2\left(u_{L}+u_{R}\right)}{u_{L}-u_{R}}
$$

where $\bar{u}=L^{-1} \int_{-L / 2}^{L / 2} u(x, t) d x$ is the instantaneous average density. The shock location fluctuates with a diffusion similar to that of a simple random walk [14] so averaging over ensembles from the same initial state gives,

$$
\left\langle\delta s^{2}\right\rangle \approx 2 \mathcal{D} t
$$


with a shock diffusion coefficient, $\mathcal{D}$, that depends on Reynolds number, shock strength, etc. Note that this expression for the variance is not accurate at very short times (due to relaxation transient from initial state) or at very long times (due to finite system size). Also note that the variance of the total mass, $\left\langle L^{2} \delta \bar{u}^{2}\right\rangle$, diffuses in the same fashion. This indicates that the diffusion of $s$ is different than other shock profile variables (e.g., center-ofmass location) that fluctuate even if $\bar{u}$ is constant.

Figure 9 shows typical results for the variance in the shock position from an ensemble of runs versus time. The hybrid algorithm is used for $p_{\rightarrow}=0.55,0.7$ and 0.8 with a time step of 0.05 integrated for two million steps; for each of these simulations the dynamic shock refinement criteria was used (see Section 4.3). The statistics were computed from 400 samples for $p_{\rightarrow}=0.55,800$ samples for $p_{\rightarrow}=0.7$, and 1200 samples for $p_{\rightarrow}=0.8$, which reflects increasing fluctuations in the shock drift at higher Reynolds number, $\operatorname{Re}_{c}$. For the intermediate case $\left(p_{\rightarrow}=0.7\right)$ we also ran the pure AERW for 400 sample over a shorter interval, demonstrating that SPDE/AERW hybrid accurately captures the behavior of the system. (Only this case was compared and the pure AERW results are for a shorter time because of the large computational expense of running the ensemble of pure particle simulations.) As expected, the shock diffusion depends on the shock strength with the strongest shock $\left(p_{\rightarrow}=0.8\right)$ exhibiting the most drift.

The most interesting feature observed in these simulations was the absence of shock diffusion in the AR hybrid using a deterministic PDE solver. This deficiency persisted even when the refined (i.e., particle) region was widened by eight cells, roughly doubling its size. The absence of shock diffusion may be, in part, due to the definition of shock location yet alternative ways of measuring the position of the shock are expected to also exhibit significantly reduced diffusion. Given that the localization of shock fronts is an important question addressed in gas dynamics simulations, the suppression of shock diffusion is a cautionary warning that the fidelity of multiscale hybrids may depend on the accuracy of the stochastic modeling.

\section{Conclusions and further work}

We have constructed a hybrid algorithm that couples an excluded random walk with a viscous Burgers' equation that represent the mean field approximation to the dynamics. The algorithm allows the random walk to be used locally to approximate the solution while modeling the system using the mean field equations in the remainder of the domain. In tests of the 


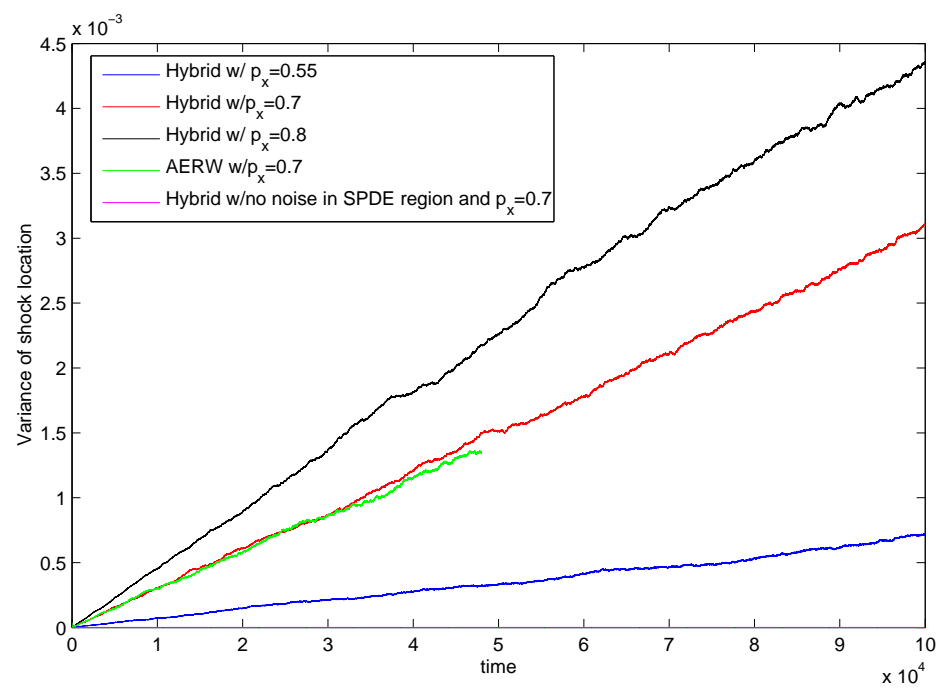

Figure 9: Variance of shock location $\left\langle\delta s(t)^{2}\right\rangle$ versus time $t$ for a deterministically steady shock $\left(u_{L}=0.1, u_{R}=0.9\right)$. Methods used are: AERW with $p_{\rightarrow}=0.7$ (green); SPDE/AERW hybrid with $p_{\rightarrow}=0.55$ (blue); SPDE/AERW hybrid with $p_{\rightarrow}=0.7$ (red); SPDE/AERW hybrid with $p_{\rightarrow}=0.8$ (black); DPDE/AERW hybrid with $p_{\rightarrow}=0.7$ with 8 cells of additional buffering (magenta).

method we have demonstrated that it is necessary to include the effect of fluctuations, represented as a stochastics flux, in the mean field equations to ensure that the hybrid preserved key properties of the system. As expected, not representing fluctuations in the continuum regime leads to a decay in the variance of the solution that penetrates into the particle region. Somewhat more surprising is that the failure to include fluctuations was shown to introduce spurious correlations of fluctuations in equilibrium simulations and for rarefactions. Even more troubling is the observation that using a deterministic PDE solver coupled to the random walk model suppresses the drift of shock location seen with the pure random walk model and with the AR hybrid using a stochastic PDE solver.

We plan to extend this basic hybrid framework to the solution of the compressible Navier Stokes equations in multiple dimension. For that extension 
we will use a Direct Simulation Monte Carlo algorithm for the microscopic model coupled to a finite difference approximation to the continuum equations, as described in [15]. The Landau-Lifshitz fluctuating hydrodynamic equations will be used to represent microscopic fluctuations at the continuum level. $[16,21]$ At present, the challenge remains to establish accurate finite difference schemes for solving these stochastic PDEs.

\section{Acknowledgment}

This work was supported by the Applied Mathematical Sciences Program of the DOE Office of Mathematics, Information, and Computational Sciences, under contract DE-AC03-76SF00098 as well as the DOE Computational Science Graduate Fellowship, under grant number DE-FG0297ER25308.

\section{References}

[1] F. J. Alexander, S. A. Janowsky, J. L. Lebowitz, and H. van Beijeren. Shock fluctuations in one-dimensional lattice fluids. Phys. Rev. E, 47:403-410, 1993.

[2] FJ Alexander, Z Cheng, SA Janowsky, and JL Lebowitz. Shock fluctuations in the two-dimensional asymmetric simple exclusion process. Journal of Statistical Physics, 68(5-6):761-785, 1992.

[3] FJ Alexander, AL Garcia, and DM Tartakovsky. Algorithm refinement for stochastic partial differential equations: I. linear diffusion. Journal of Computational Physics, 182(1):47-66, 2002.

[4] FJ Alexander, AL Garcia, and DM Tartakovsky. Algorithm refinement for stochastic partial differential equations. AIP CONFERENCE PROCEEDINGS, 663:915-922, 2003.

[5] FJ Alexander, AL Garcia, and DM Tartakovsky. Algorithm refinement for stochastic partial differential equations: II. correlated systems. Journal of Computational Physics, 207:769-787, 2005.

[6] M. J. Berger and P. Colella. Local adaptive mesh refinement for shock hydrodynamics. 82(1):64-84, 1989.

[7] M. J. Berger and J. Rigoutsos. An algorithm for point clustering and grid generation. 21:1278-1286, 1991. 
[8] L Bertini, N Cancrini, and G Jona-Lasinio. The stochastic burgers equation. Comm. Math. Phys., 165(2):211-232, 1994.

[9] L Bertini and G Giacomin. Stochastic burgers and kpz equations from particle systems. Communications in Mathematical Physics, 183(3):571-607, 1997.

[10] BM Boghosian and CD Levermore. A cellular automaton for burgers' equation. Complex Systems, 1:17, 1987.

[11] L Brieger and E Bonomi. A stochastic lattice gas for burgers' equation: A practical study. Journal of Statistical Physics, 69(3-4):837-855, 1992.

[12] H. J. Bussemaker and M. H. Ernst. Microscopic theory for long-range spatial correlations in lattice gas automata. Phys. Rev. E, 53:5837-5851, June 1996.

[13] R. Delgado-Buscalioni and P. V. Coveney. Continuum-particle hybrid coupling for mass, momentum, and energy transfers in unsteady fluid flow. Physical Review E, 67(4):046704-+, 2003.

[14] PA Ferrari and LRG Fontes. Shock fluctuations in the asymmetric simple exclusion process. Probability Theory and Related Fields, 99(2):205-319, 1994.

[15] AL Garcia, JB Bell, WY Crutchfield, and BJ Alder. Adaptive mesh and algorithm refinement using direct simulation monte carlo. Journal of Computational Physics, 154(1):134-155, 1999.

[16] AL Garcia, M Malek Mansour, G Lie, and E Clementi. Numerical integration of the fluctuating hydrodynamic equations. Journal of Statistical Physics, 47:209, 1987.

[17] PL Garrido, JL Lebowitz, C Maes, and H Spohn. Long-range correlations for conservative dynamics. Phys. Rev. A, 42(4):1954-1968, 1990.

[18] C. Haselwandter and D. D. Vvedensky. Fluctuations in the lattice gas for Burgers' equation. Journal of Physics A Mathematical General, 35:L579-L584, 2002.

[19] F. Hayot and C. Jayaprakash. From scaling to multiscaling in the stochastic Burgers equation. Physical Review E, 56(4):4259-4262, 1997. 
[20] S. A. Janowsky and J. L. Lebowitz. Finite-size effects and shock fluctuations in the asymmetric simple-exclusion process. Physical Review A, 45:618-625, January 1992.

[21] LD Landau and EM Lifshitz. Fluid Mechanics, volume 6 of Course of Theoretical Physics. Pergamon, 1959.

[22] J. L. Lebowitz, E. Orlanndi, and E. Presutti. Convergence of stochastic cellular automation to burger's equation: fluctuations and stability. Physica D, 33(1-3):165-188, 1988.

[23] T.M. Liggett. Interacting Particle Systems. Springer-Verlag, New York, 1985.

[24] D. Mitra, J. Bec, R. Pandit, and U. Frisch. Is Multiscaling an Artifact in the Stochastically Forced Burgers Equation? Physical Review Letters, 94(19):194501, 2005.

[25] E Moro. Hybrid method for simulating front propagation in reaction-diffusion systems. PHYSICAL REVIEW E, 69(6):060101, 2004 .

[26] M Plapp and A Karma. Multiscale finite-difference diffusion-monte-carlo method for simulating dendritic solidification. Journal of Computational Physics, 165(2):592-619, 2000.

[27] W Ren and W E. Heterogeneous multiscale method for the modeling of complex fluids and micro-fluidics. Journal of Computational Physics, 204(1):1-26, 2005.

[28] TP Schulze, P Smereka, and W E. Coupling kinetic monte-carlo and continuum models with application to epitaxial growth. Journal of Computational Physics, 189(1):197-211, 2003.

[29] H. Spohn. Long range correlations for stochastic lattice gases in a non-equilibrium steady state. Journal of Physics A Mathematical General, 16:4275-4291, 1983.

[30] H. Spohn. Large Scale Dynamics of Interacting Particles. Springer-Verlag, New York, 1991.

[31] P Stinis. A hybrid method for the inviscid burgers equation. Discrete And Continuous Dynamical Systems, 9(4):793-799, 2003. 
[32] Quanhua Sun, Iain D. Boyd, and Graham V. Candler. A hybrid continuum/particle approach for modeling subsonic, rarefied gas flows. J. Comput. Phys., 194(1):256-277, 2004.

[33] Schwartzentruber TE and Boyd ID. A hybrid particle-continuum method applied to shock waves. Journal of Computational Physics, to appear, 2006.

[34] M Tysanner and AL Garcia. Non-equilibrium behavior of equilibrium reservoirs in molecular simulations. International Journal of Numerical Methods in Fluids, (to appear): , 2005.

[35] E.G. Flekkoy ; J. Feder ; G. Wagner. Coupling particles and fields in a diffusive hybrid model. Physical Review E, 64:066302, 2001.

[36] T Werder, JH Walther, and P Koumoutsakos. Hybrid atomisticcontinuum method for the simulation of dense fluid flows. Journal of Computational Physics, 205(1):373-390, 2005.

[37] HS Wijesinghe, R Hornung, AL Garcia, and NG Hadjiconstantinou. Three-dimensional hybrid continuum-atomistic simulations for multiscale hydrodynamics. Journal of Fluids Engineering, 126:768-77, 2004 . 